\title{
Tunable, low frequency microwave generation from AWG based closely-spaced dual-wavelength single-longitudinal-mode fibre laser
}

H. Ahmad

harith@um.edu.my

A. A. Latif

J. M. Taib

S. W. Harun
Photonics Research Centre (Department of Physics), University of Malaya, 50603 Kuala Lumpur, Malaysia

Visiting Professor, Nanotechnology Research Alliance, Universiti Teknologi Malaysia, Skudai, 81310 Johor, Malaysia

Photonics Research Centre (Department of Physics), University of Malaya, 50603 Kuala Lumpur, Malaysia

Photonics Research Centre (Department of Physics), University of Malaya, 50603 Kuala Lumpur, Malaysia

Photonics Research Centre (Department of Physics), University of Malaya, 50603 Kuala Lumpur, Malaysia

Stable, closely-spaced Dual-Wavelength Fibre Lasers (DWFLs) have high potential for applications such as Radio-over-Fibre and optical sensing. In this work, a DWFL using two Arrayed Waveguide Gratings (AWGs) to generate a closely-spaced dual-wavelength output is proposed and demonstrated. A $1 \mathrm{~m}$ long highly doped Leikki Er80-8/125 Erbium Doped Fibre (EDF) is used as the linear gain medium, while two AWGs are used to generate the closely-spaced lasing wavelengths. A Mach-Zehnder modulator, driven at $180 \mathrm{kHz}$, splits the closely spaced wavelengths into two clearly defined wavelengths, while a $7 \mathrm{~cm}$ long un-pumped Leikki Er80-8/125 EDF and sub-ring cavity is used to suppress unwanted side-modes. Close spacing of between 0.01 to $0.03 \mathrm{~nm}$ are obtained, with beating frequencies of between 1.4 to 3.2 $\mathrm{GHz}$. The output is highly stable, with almost no fluctuations over the test period.

[DOI: http://dx.doi.org/10.2971/jeos.2013.13038]

Keywords: Closely-spaced, frequency beating, AWG, modulation

\section{INTRODUCTION}

Dual-Wavelength Fibre Lasers (DWFLs) have gained much attention due to its wide potential for application in a variety of tasks. DWFLs are particularly useful in optical sensing such as signal sources, such as terahertz sources, Differential Absorption LIDAR (DIALs) sources and sources for chemical and biological applications. They also find significant use in a variety of communication applications, such as wavelength conversion as well as in Radio-over-Fibre (RoF) systems as microwave and millimetre wave generators.

Fibre based DWFLs are highly advantageous over other optical approaches due to their relatively smaller size and lower complexity, as well as having a typically lower cost. Most fibre-based DWFL designs employ the Erbium Doped Fibre (EDF) as the primary gain medium in their design. However, the homogenous gain broadening of the EDF's output, which is due to the inherent characteristics of the erbium ions, typically results in unstable lasing wavelengths due to modecompetition and mode-hopping. There have been reports of using the Semiconductor Optical Amplifier (SOA), an inhomogenously gain broadened media to overcome the effects of mode-competition and mode-hopping [1]-[3], although at the cost of higher noise and increased power losses in the system. However, there is still significant interest to use the EDF as the gain medium, as its behaviour is well documented and under- stood. Various methods have been put forward to overcome the homogenous gain broadening effects in the EDF, including cooling the EDF with liquid nitrogen $[4,5]$ or using specially designed elliptical EDFs [6] which are very complex and not practical. Other approaches have also been explored, including the use of polarization maintaining Fibre Bragg Gratings (FBGs) for selecting and isolating specific wavelengths [7], frequency shifters [8], Distributed Fibre Bragg (DFB) laser sources [9], the Four-Wave-Mixing effect [10], optical injection Fabry-Perot lasers [11], cavity loss control [12] and even dualring fibre lasers [13]. These approaches, while less complex, are still difficult to practically implement.

From the above, it can be easily concluded that EDF based DWFLs will be the best approach for microwave or millimetre-wave generation. The main interest would be to generate microwave signals in the lower frequency region, which will not require expensive detection equipment. Of late, there have been reports of the use of a tunable and switchable DWFL for generating microwave signals, with an output at $9.8 \mathrm{GHz}$ [14]. Using a similar DWFL approach, there is also a report of microwave generation as low as $3.4 \mathrm{GHz}$ [15]. In this work, the authors demonstrate a compact DWFL based on a highly doped $1 \mathrm{~m}$ long Leikki EDF together with two Arrayed Waveguide Gratings (AWGs) capable of 


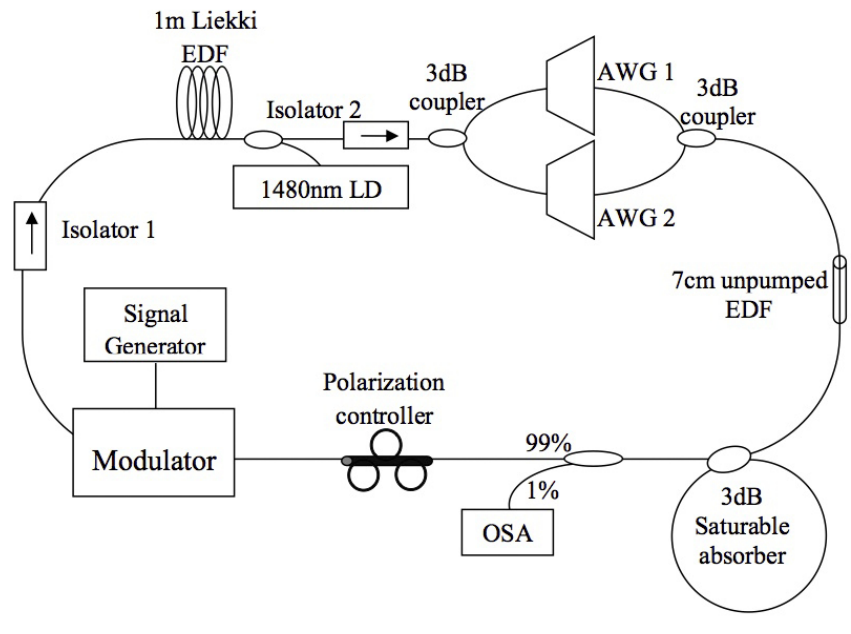

FIG. 1 Schematic configuration showing the various components for generating the closely-spaced DWFL using two AWGs.

generating dual-wavelength outputs with substantially narrower channel spacings, thus allowing for the generation of microwave signals much lower than those mentioned above. The lasing wavelengths operate in a Single-LongitudinalMode, as confirmed by a single peak from the beating of the (SLM) dual-wavelength output when observed in the radio frequency spectrum. This is, to the knowledge of the author, the first report of microwave generation from a DWFL at this low a frequency with a modulated output.

\section{EXPERIMENTAL SETUP}

Figure 1 shows the setup of the proposed AWG-based DWFL that can provide closely spaced dual-wavelength outputs which will lead to a lower frequency beating. It consists of a 1 m highly doped Liekki EDF (Er80-8/125), with absorption of 45 and $80 \mathrm{~dB} / \mathrm{m}$ at 1400 and $1530 \mathrm{~nm}$ respectively as the linear gain medium. The EDF is pumped in a counter-propogating configuration by a $1480 \mathrm{~nm}$ Laser Diode (LD), operating at $340 \mathrm{~mW}$, through a 1480/1550 nm Wavelength Division Multiplexer (WDM). The ASE spectrum generated by the pumped EDF will be emitted at both ends of the EDF. As such, two optical isolators (Isolator 1 and Isolator 2) are placed in the cavity, before and after the EDF to ensure uni-directional operation in the clockwise direction. The ASE propagating through Isolator 2 will now enter a $3 \mathrm{~dB}$ coupler, where it is split into two even portions and directed into two AWGs, designated AWG1 and AWG2. The AWGs are of similar specifications, with a wavelength accuracy of accuracy of $\pm 0.04 \mathrm{~nm}$ as well as a $1 \mathrm{~dB}$ bandwidth and $3 \mathrm{~dB}$ bandwidth of about $0.2 \mathrm{~nm}$ and $0.4 \mathrm{~nm}$ respectively.

This is then recombined with a second $3 \mathrm{~dB}$ coupler, which is in turn connected to a $7 \mathrm{~cm}$ long unpumped EDF of the same type as described above.The short, un-pumped EDF acts as a saturable absorber to suppress the unwanted, weaker modes and allow only the dominant wavelengths to oscillate in the cavity.

The output of the unpumped EDF is then connected to a $1.55 \mathrm{~m}$ long sub-ring cavity with the specific purpose of suppresing the side modes, based on the Vernier effect $[16,17]$. The output of the sub-ring cavity is connected to the common port of a 99:1 fused tap coupler, with $1 \%$ of the propagating signal being extracted for further analysis, while the remaining $99 \%$ is allowed to continue propagating within the ring cavity. The $99 \%$ port is connected to a Polarization Controller (PC) and subsequently to a MachZehndermodulator (JDS Uniphase OC-192), which has a bias voltage of $5.7 \mathrm{~V}$ and is driven by a $180 \mathrm{KHz}$ transmission by a Synthesized Signal Generator (SSG) from Anritsu. Where the un-pumped EDF and the sub-ring cavity act to suppress unwanted side-modes, the modulator splits the two propagating waves from the AWG (which until this point have overlapped) into two distinct waves. The generation of the dual-wavelength output is attributed to birefringence in the cavity. This essentially gives the cavity two optical path lengths, and such allows for two dominant wavelengths to oscillate in the cavity [18]. The dual-wavelength output generated now continues to travel to Isolator 2 and back to the $1 \mathrm{~m}$ long Leikki EDF, thus completing the optical cavity. Optical analysis of the generated signal is done using a Yokogawa AQ6370B Optical Spectrum Analyzer (OSA) with $0.02 \mathrm{~nm}$ resolution. In order to measure the lasing profile of the output, the OSA in the setup is replaced by a LeCroy oscilloscope with a $500 \mathrm{MHz}$ bandwith together with a photodetector. Similarly, the photodiode and oscilloscope are replaced by a Radio Frequency Spectrum Analyzer (RFSA) to observe frequency beating measurements.

\section{RESULTS AND DISCUSSION}

Figure 2(a) shows the DWFL with a spacing of $0.03 \mathrm{~nm}$, which is equal to $3.2 \mathrm{GHz}$ spacing from the two AWGs as shown in the setup of Figure 1. The splitting of the dual-wavelength is observed when the fibre laser is modulated with a $180 \mathrm{KHz}$ sinusoidal pulse from the SSG. The two channels from the two AWGs have about the same wavelength output. Without the modulators, it will be difficult to split these two wavelengths. By carefully controlling the PC, dual-wavelength outputs with a $0.03 \mathrm{~nm}$ spacing is obtained.

Figure 2(b) shows the modulated output pulse train from the DWFL output with a nearly similar repetition rate as that of the modulator. The pulse train is a combination of the dualwavelength output of both shorter and longer wavelength of $1530.48 \mathrm{~nm}$ and $1530.51 \mathrm{~nm}$ respectively.

Figure 3(a) shows the spectrum for each wavelength from the closely-spaced dual-wavelength fibre laser as taken from the OSA. The PC used to select one wavelength out of the two by controlling the polarization state of the laserinside the cavity. It can be observed from Figure 3(a) that the dual-wavelength fibre laser is succesfully divided be selecting one lasing wavelength at a time through carefull adjustment of the PC. The wavelength of shorter and longer wavelength are $1530.48 \mathrm{~nm}$ and $1530.51 \mathrm{~nm}$ respectively as in the earlier case.

The pulse train in the time domain is taken from the oscilloscope, as depicted in Figure 3(b), showing the pulse train for both the shorter and the longer wavelength outputs. The 

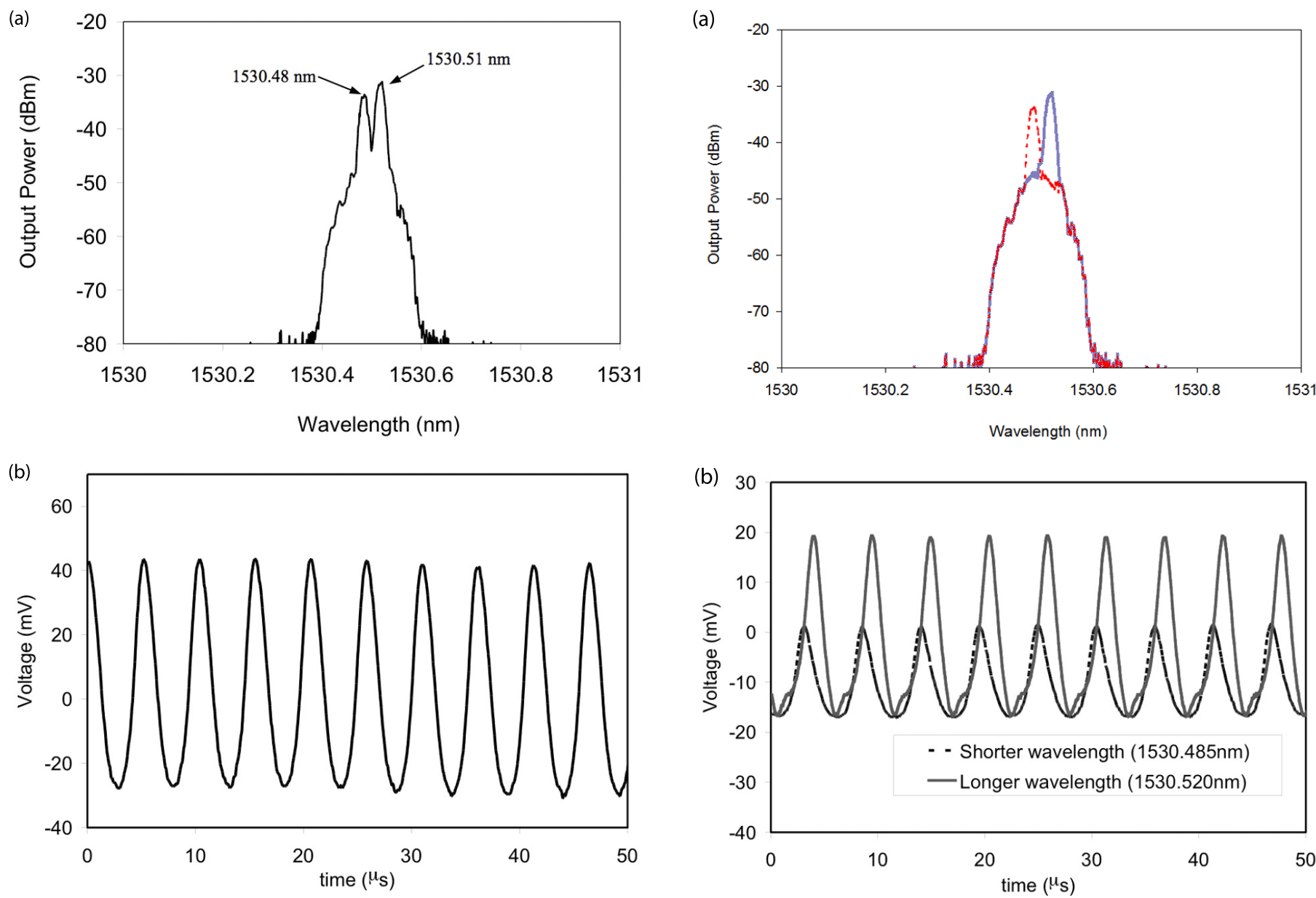

FIG. 2 (a) spectrum of the closely-spaced dual-wavelength fibre laser using two AWGs and (b) the pulse train as observed from the oscilloscope for the closely-spaced dualwavelength output, which has a repetition rate of about $180 \mathrm{kHz}$.

repetition rate for both the shorter wavelength and the longer wavelength are approximately $180 \mathrm{KHz}$, with the pulse of the longer wavelength having a higher amplitude compared to the pulse of the shorter wavelength.

On top of this, narrower dual-wavelength spacing can be achieved by selecting channels from the two AWGs, with wavelengths such as $1530.44 \mathrm{~nm}$ and $1530.45 \mathrm{~nm}$, as measured giving a dual-wavelength spacing of approximately $0.01 \mathrm{~nm}$. This is shown in Figure 4(a), with a frequency beating of $1.4 \mathrm{GHz}$ as observed from the RFSA. Figure 4(b) shows the corresponding optical spectrum as obtained from the OSA, with a spacing of $0.01 \mathrm{~nm}$. In the same manner, a beating frequency of $2.5 \mathrm{GHz}$ is obtained for a wavelength spacing of $0.02 \mathrm{~nm}$, as shown in Figures 4(c) and 4(d) respectively, while a beating frequency of $3.2 \mathrm{GHz}$ is obtained for a wavelength spacing of $0.03 \mathrm{~nm}$, as shown in Figures 4(e) and 4(f). From these figures, it can be inferred that due to the single peak in the RF spectrum, that the two wavelengths are being operated as SLMs.

Figure 5 shows the baseband data arises from the modulation pulse of the SSG. This modulation pattern can only be observed when taken in a small range, from 0 to $2 \mathrm{MHz}$, and as can be seen from the figure a constant gap of $180 \mathrm{KHz}$ between each adjacent peak is obtained. This confirms that the modulation of the pulse is a result of the modulator, and not other

FIG. 3 (a) the spectrum of the fibre laser with the modulation technique and (b) the pulse train observed by a photodetector with an oscilloscope for the closely-spaced dual-wavelength output.
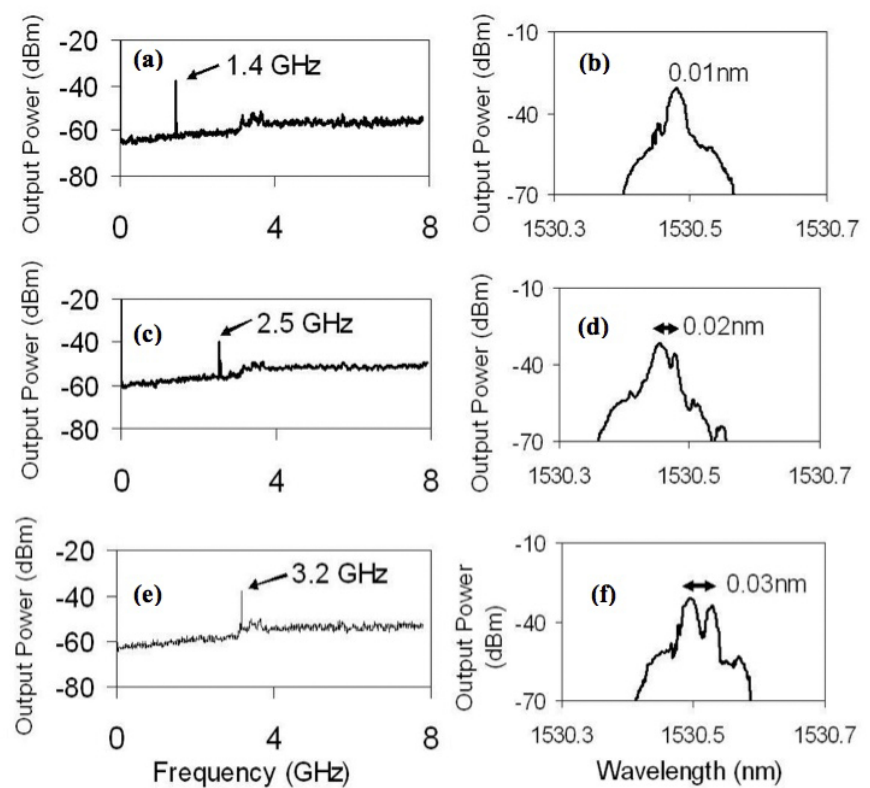

FIG. 4 The frequency of the closely-spaced dual-wavelength output (a), (c) and (e) taken from the RFSA and the (b), (d) and ( $f$ ) the respective dual-wavelength spacing spectra taken from the OSA.

by other optical phenomena. As such, the stabilization of the dual-wavelength output is a result of the 'pulse splitting' that 


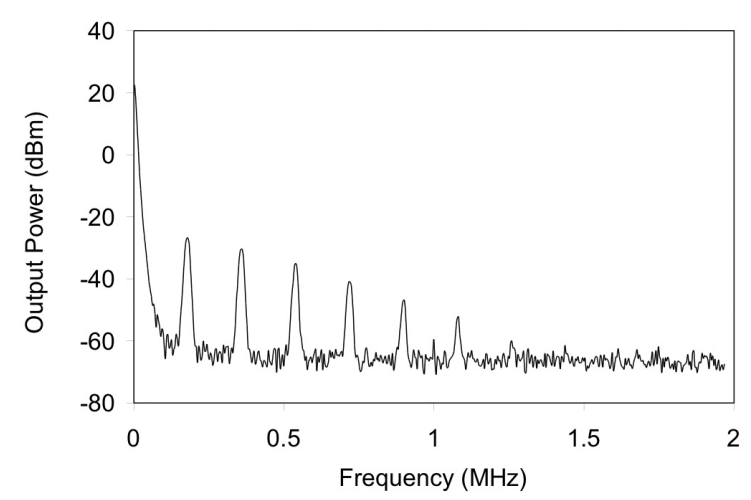

FIG. 5 Baseband frequency taken from the RFSA.

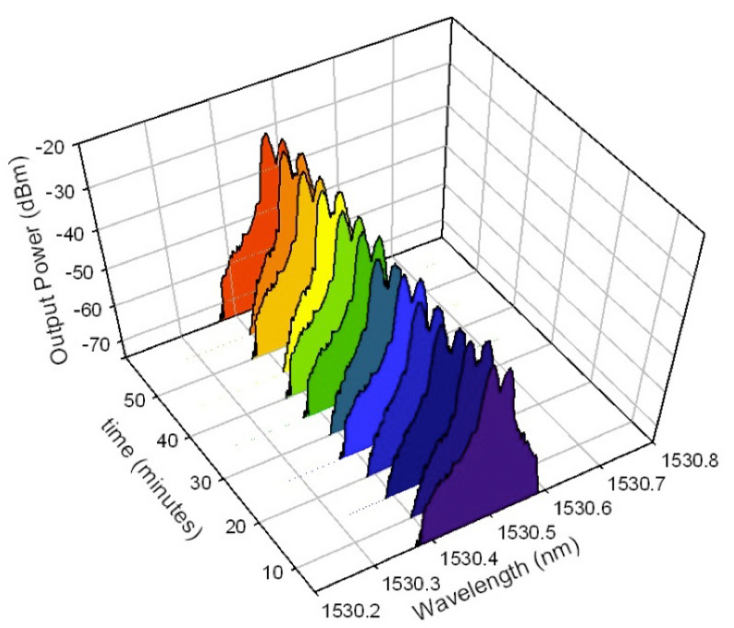

FIG. 6 Stability performance of the DW-close spacing SLM fibre laser by using two AWGS configuration.

arises from the modulation of the propagating wavelengths in the laser cavity.

Figure 6 shows the stability of the closely-spaced dualwavelength fibre laser over an hour of operation at intervals of 5 minutes. The spacing of the wavelength is $0.03 \mathrm{~nm}$, equivalent to $3.2 \mathrm{GHz}$.

From the figure, it can be seen that the output of the system is highly stable, with only minor fluctuations in the spacing and power of the laser. Thus, the system can be used as a highly stable dual-wavelength laser source, with a close spacing the generation of microwave signals in the low frequency region, which in turn can find many useful applications such as RoF and sensor applications.

\section{CONCLUSION}

In this work, a DWFL using two AWGs to generate a closelyspaced output is proposed and demonstrated. The DWFL uses a $1 \mathrm{~m}$ long highly doped Leikki Er80-8/125 EDF as the primary gain medium, while the two AWGs are used to generate the dual-wavelength output. A $7 \mathrm{~cm}$ long un-pumped Er808/125 EDF, together with a sub-ring cavity is used to suppress unwanted side-modes, while a Mach-Zehnder modulator driven at $180 \mathrm{kHz}$ is used to split the oscillating wave- lengths such that two, distinct lasing wavelengths are obtained. Lasing wavelengths with spacing of $0.01,0.02$ and $0.03 \mathrm{~nm}$ (corresponding to beating frequencies of 1.4, 2.5 and $3.2 \mathrm{GHz}$ ) are obtained. The generated output is highly stable, with only minor fluctuations in spacing and power, making it highly suitable for applications such as Radio-over-Fibre and sensors.

\section{ACKNOWLEDGEMENTS}

We would like to thank the University of Malaya for providing the funding for this project under the UMRG Grant (RG14312AET).

\section{References}

[1] S. Pan, X. Zhao, and C. Lou, "Switchable single-longitudinal-mode dual-wavelength erbium-doped fibre ring laser incorporating a semiconductor optical amplifier," Opt. Lett. 33, 764-766 (2008).

[2] H. Ahmad, M. Z. Zulkifli, A. A. Latif, K. Thambiratnam, and S. W. Harun, "Dual-wavelength fibre laser with tunable channel spacing using an SOA and dual AWGs," J. Mod. Opt. 56, 1768-1773 (2009).

[3] M. Tang, H. Minamide, Y. Wang, T. Notake, S. Ohno, and H. Ito, "Tunable terahertz-wave generation from DAST crystal pumped by a monolithic dual-wavelength fibre laser," Opt. Express 19, 779-786 (2011).

[4] S. Yamashita, and K. Hotate, "Multiwavelength erbium-doped fibre laser using intracavity etalon and cooled by liquid nitrogen," Electron. Technol. Lett. 32, 1298-1299, (1996).

[5] N. Park, and P. F. Wysoncki, "24-line multiwavelength operation of erbium doped fibre ring laser," IEEE Photon. Technol. Lett. 8, 1459-1461 (1996).

[6] G. Das, and J. W. Y. Lit, "L-band multiwavelengthfibre laser using elliptical fibre," IEEE Photon. Technol. Lett. 14, 606-608 (2002).

[7] S. Feng. 0. Xu, S. Lu, T. Ning, and S. Jian, "Switchable singlelongitudinal-mode dual-wavelength erbium-doped fibre ring laser based on polarization-maintaining fibre Bragg grating incorporating saturable absorber and feedback loop mirror," 0pt. Commun. 282, 825-831 (2009).

[8] A. Bellemare, M. Karasek, M. Rochette, S. L. Rochelle, and M. Tetu, "Room temperature multifrequency erbium-doped fibre lasers anchored on the ITU frequency grid," J. Lightwave Technol. 18, 825-832 (2000).

[9] J. Sun, Y. Dai, X. Feng, Y. Zhang, and S. Xie, "Stable dualwavelength DFB fibre laser with separate resonant cavities and its application in tunable microwave generation," IEEE Photon. Technol. Lett. 18, 2587-2589 (2006).

[10] M. P. Fok, and C. Shu, "Tunable dual-wavelength erbium-doped fibre laser stabilized by four-wave mixing in a $35 \mathrm{~cm}$ highly nonlinear bismuth-oxide fibre," Opt. Express 15, 5925-5930 (2007).

[11] C. H. Yeh, C. W. Chow, F. Y. Shih, C. H. Wang, Y. F. Wu, and S. Chi, " Tunable dual-wavelength fibre laser using opticalinjection Fabry-Perot laser," IEEE Photon. Technol. Lett. 21, 125-127 (2009).

[12] H. Ahmad, M. Z. Zulkifli, A. A. Latif, and S. W. Harun, "Tunable dual-wavelength fibre laser incorporating AWG and optical channel selector by controlling the cavity loss," Opt. Commun. 282, 4771-4775 (2009). 
[13] C. H. Yeh, "Stabilized dual-wavelength erbium-doped dual-ring fibre laser," Opt. Express 15, 13844-13848 (2007).

[14] F. Wang, X. Zhang, Y. Zhang, and E. Xu, "A Tunable and Switchable Single-longitudinal-mode Dual-wavelength Fibre Laser for Microwave Generation," in Proceedings of Communications and Photonics Conference and Exhibition (ACP), 728-729 (SPIE, Shanghai, 2010).

[15] Y. Yao, X. Chen, Y. Dai, and S. Xie, "Dual-wavelength erbiumdoped fibre laser with a simple linear cavity and its application in microwave generation," IEEE Photon. Technol. Lett. 18, 187-189 (2006).
[16] C. Lee, Y. Chen, and S. Liaw, "Single-longitudinal-mode fiber laser with a passive multiple-ring cavity and its application for video transmission," Opt. Lett. 23, 358-360 (1998).

[17] X. Yang, L. Zhan, and Y. Xia, “High-power single-longitudinal-mode fiber laser employing two Sagnac loop filters," Opt. Eng. 47, 065001 (2008).

[18] J. B. Schlager, S. Kawanishi, and M. Saruwatari "Dual wavelength pulse generation using mode-locked erbium-doped fibre ring laser," Electron. Lett. 27, 2072-2073 (1991). 